\title{
Method of analysis of geomagnetic data based on wavelet transform and threshold functions
}

\author{
O. Mandrikova ${ }^{1,2}$, I. Solovev ${ }^{1,2}$, S. Khomutov ${ }^{1}$, K. Arora ${ }^{3}$, L. Manjula ${ }^{3}$, P. Chandrasekhar ${ }^{3}$ \\ ${ }^{1}$ Institute of Cosmophysical Research and Radio Wave Propagation FEB RAS, 684034, Paratunka, Russia \\ ${ }^{2}$ Kamchatka State Technical University, 683003, Petropavlovsk-Kamchatsky, Russia \\ ${ }^{3}$ CSIR-National Geophysical Research Institute, 500007, Hyderabad, India
}

\begin{abstract}
The suggested method is aimed at studying the dynamics of the magnetospheric current systems during magnetic storms. The method is based on algorithmic solutions for processing of geomagnetic field variations, detection of local increases in geomagnetic disturbance intensity and estimation of their dynamic characteristics. Parameters of the algorithms allow us to evaluate the characteristics of small-scale local features emerging during geomagnetic activity slight increases and large-scale variations observed during magnetic storms. To evaluate the method, geomagnetic data from the stations located in the north-east of Russia and equatorial India were used. The method testing showed the possibility to apply it for the detection of pre-storm anomalous effects in geomagnetic data.
\end{abstract}

Keywords: Wavelet transform; geomagnetic data processing; magnetic storm

\section{Introduction}

The work is devoted to the creation of methodical and software means for the analysis of recorded geomagnetic data. At present, theoretical and experimental bases of construction of systems for data processing and analysis are intensively developing, in particular in geophysics (for example, http://www.cosmos.ru/magbase; http://matlab.izmiran.ru/magdata/; https://www.ngdc.noaa.gov/; http://smdc.sinp.msu.ru/). It is caused by the increase of human society demands in automation of data flow processing. The subjects of this investigation are complex dynamic processes in the Earth magnetosphere and ionosphere determined by the phenomena and processes of solar origin. Solar activity impact on the Earth magnetosphere has quite a complicated character. Many aspects of it are still under-investigated [1]. As long as the state of magnetosphericionospheric system is an important factor of space weather which affects many aspects of our life, works in this area are of high scientific interest [1].

The Earth magnetic field variations reflect different geophysical processes in the Earth near space. During magnetic storms they contain uneven local features occurring at random times and carrying important information on the processes in the magnetosphere [2-14]. Traditional methods for data analysis applying basic models of time series, different techniques of smoothing and Fourier analysis methods are not effective enough to investigate fast unsteady processes. As it was noted in the papers [15-18], they do not allow one to identify thin local features characterizing short-period oscillations during increased geomagnetic activity and to estimate their dynamic characteristics before and during storms. At present, modern mathematic methods and technologies are intensively developing in this area [3-9, 14, 18-25]. Based on Data Mining application in order to improve the processes of geophysical data recording and organization of world data centers, methods for automation of expert work in this area have been developed (creation of so called "artificial experts") to solve geomagnetic data analysis problems, to detect noise at the stage of their preliminary processing, to identify anomalies during magnetic storms, to process magnetograms etc. [19-25]. To solve these tasks, the authors apply of the papers [9] a new approach, «discrete mathematical analysis» (DMA), which includes fundamental notions of mathematical analysis and modern approaches based on L. Zadeh's logic. A group of scientists from India (Kaleekkal Unnikrishnan) developed a technique for modeling of geomagnetic field variations for lowlatitudinal stations. It is based on neural networks. The developed approach allowed them to improve the quality of the forecasting technique for magnetic storms (in $86 \%$ of cases) in comparison to the nearest method based on logistic regressive model obtained in 2005 (in 77\% of cases) [23]. A group of scientists from Egypt (Space Weather Center, Faculty of Science, Helwan University, Cairo, Egypt) suggested to apply neural networks to predict the time of interplanetary shock wave propagation [24]. Based on the neural networks, the authors [25] suggested an algorithm for interplanetary magnetic field data processing and Dst-index calculation. The authors of that paper suggest an approach based on the combination of neural networks with wavelet transform and show the efficiency of joint application of mathematical apparatus data in comparison with a neural network in the problems of analysis of natural time series with complicated structures [26-29], in particular, for geomagnetic data analysis $[28,29]$. It is shown in these papers that wavelet transform allows us to investigate the data structure in detail and to detect informative components which, in their turn, improve the procedure of neural network training and its performance efficiency. At present time, wavelet transform is widely used in the problems of analysis of the Earth magnetic field variations [2-5, 10, 14]. In the paper [2] wavelet transform is applied to investigate the relations between short-period oscillations of the geomagnetic field, solar wind parameters and interplanetary field during geomagnetic storms. Based on the wavelet transform, we solve such problems as denoising and elimination of a periodic component from geomagnetic field variations which is caused by the Earth rotation [4]. Applying the discrete wavelet transform, the authors of the paper [3] suggested an algorithm for automatic detection of magnetic storm initial stage periods. On the basis of the analysis of geomagnetic field variation wavelet spectrum, a method for forecast of strong geoeffective solar flares was proposed [13]. In 
Image Processing, Geoinformation Technology and Information Security / O. Mandrikova et al.

terms of wavelets, the authors of this paper suggested a new model of geomagnetic field variations [14, 30] and developed automatic algorithms do detect calm diurnal variation and to estimate disturbance intensities [30]. This approach allowed us to automate the procedure for calculation of geomagnetic activity index $\mathrm{K}$, close to $\mathrm{J}$. Bartels method, and to decrease the calculation error in comparison to the current methods [30]. In this paper we continue the investigation in this direction where a special emphasis is placed on the development of calculation solutions to detect and to estimate short-time anomalous increases in geomagnetic disturbance intensity which may occur before magnetic storms and have applied significance. The important thing in this approach is the possibility to apply the geomagnetic field data recorded on the ground, the analysis methods of which may significantly contribute to the current forecast methods. Taking into account incomplete prior knowledge on the dynamics of magnetospheric current systems and the limited scope of the obtained information on the processes in the near Earth space, noises, possible equipment failures etc., successful solution of the problem of space weather forecast requires a complex of methods and technologies. The confirmation of it is the large number of papers and scientific groups which aim their efforts at creating methods for recognition and classification of the effects in geophysical observation time series with applications in space weather problems.

\section{Description of the method}

In the papers $[14,30]$ the authors propose geomagnetic field variation representation based on multiscale wavelet decompositions:

$$
f_{0}(t)=\sum_{n} c_{-6, n} \varphi_{-6, n}(t)+\sum_{j \in D} \sum_{n} d_{j, n} \Psi_{j, n}(t)+\sum_{j \notin D} \sum_{n} d_{j, n} \Psi_{j, n}(t)=f_{\text {trend }}(t)+f_{\text {dist }}(t)+e(t),
$$

where $\Psi_{j}=\left\{\Psi_{j, n}\right\}_{n \in Z}$ is the wavelet-basis, $\varphi_{j}=\left\{\varphi_{j, n}\right\}_{n \in Z}$ is the basis, obtained from a scaling function, coefficients $c_{j, n}$ and $d_{j, n}$ are defined from the equations: $c_{j, n}=\left\langle f, \varphi_{j, n}\right\rangle, d_{j, n}=\left\langle f, \Psi_{j, n}\right\rangle, D$ is a set of indices of the disturbed components, $j$ is the scale, the inferior index «0» denotes that the initial discrete data belong to a domain of scale «0».

Component $f_{\text {trend }}(t)=\sum_{n} c_{-6, n} \varphi_{-6, n}(t)$ describes the undisturbed level of the horizontal component of the Earth magnetic field during quiet geomagnetic field, and the component $f_{d i s t}(t)=\sum_{j \in D} g_{j}(t)$ where $g_{j}(t)=\sum_{n} d_{j, n} \Psi_{j, n}(t)$ describes perturbations, arising during increasing geomagnetic activity. Component $e(t)=\sum_{j \notin D} \sum_{n} d_{j, n} \Psi_{j, n}(t)$ is the noise.

Minimizing the errors in the class of orthonormal functions, the Daubechies basis of order 3 was determined as the wavelet basis [30].

The set of indices $D$ can be determined on the basis of the following criteria $[14,30]$ :

$$
j \in D, \text { if } m\left(A_{j}^{v}\right)>m\left(A_{j}^{k}\right)+\varepsilon,
$$

where $m$ is the sample mean, $v$ is the index of disturbed field variation, $k$ is the index of calm field variation, and $\varepsilon$ is a positive number.

Assuming that $A_{j}^{v}$ and $A_{j}^{k}$ are normally distributed with mean $\mu^{v}, \mu^{k}, \mu^{v}>\mu^{k}$ and variances $\sigma^{2, v}, \sigma^{2, k}$, it is possible to estimate $\varepsilon_{j}$ as $\hat{\varepsilon_{j}}=x_{1-a / 2} \frac{\sigma_{j}^{k}}{\sqrt{n^{k}}}$, where $\sigma_{j}^{k}$ is the variance of the greatest wavelet coefficients (for scale $j$ ) for quiet days (this variance is determined as a result of multiple measurements); $x_{1-a / 2}$ is the $1-a / 2$ quintile of the standard normal distribution; $n^{k}$ is the number of analyzed quiet-field variations. If $a=0.1$, the confidence probability is $1-a / 2=0.95$, the quintile is $x_{1-a / 2}=1.96$, and $\varepsilon_{j}=1.96 \frac{\sigma_{j}}{\sqrt{n}}$.

The measure of geomagnetic disturbance of the component $g_{j}(t)$ on the scale $j$ is $[14,30]$ :

$$
A_{j}=\max _{n}\left(\left|d_{j, n}\right|\right) \text {. }
$$

Taking into account that the component $f_{\text {dist }}(t)=\sum_{j \in D} g_{j}(t)$, where $g_{j}(t)=\sum_{n} d_{j, n} \Psi_{j, n}(t)$ describes the disturbances (see relation (1)), and the equivalence of discrete and continuous wavelet decompositions, in order to obtain more detailed information on the properties of the function $f$ under analysis, continuous wavelet transform may be applied [31, 32]

$$
\left(W_{\Psi} f\right)(b, a):=|a|^{-1 / 2} \int_{-\infty}^{\infty} f(t) \Psi\left(\frac{t-b}{a}\right) d t, \Psi \text { is the wavelt, } f \in L^{2}(R), a, b \in R, a \neq 0,
$$


In this case, when a scale $a$ vanishes, the wavelet coefficients $\left(W_{\Psi} f\right)(b, a)$ characterize the local properties of the function $f$ in the vicinity of the instant time $t=b[31,32]$.

Following the relation (3) as a measure of geomagnetic disturbance intensity, it is logical to consider the wave coefficient amplitude

$$
i_{b, a}=\left|\left(W_{\Psi} f_{b, a}\right)\right|
$$

The intensity of field multi-scale disturbances at an instant time $t=b$ is estimated on the basis of the value [14, 30]

$$
I_{b}=\sum_{a}\left(W_{\Psi} f_{b, a}\right)
$$

In the case of field positive disturbances (current variation increase relatively the characteristic level), $I_{b}$ value is positive. In the case of field negative disturbances (variation decrease relatively the characteristic level), $I_{b}$ value is negative.

To distinguish the periods of increased geomagnetic activity, the following threshold function is applied:

$$
P_{T_{a}}\left(W_{\Psi} f_{b, a}\right)=\left\{\begin{array}{l}
W_{\Psi} f_{b, a}, \text { если }\left(W_{\Psi} f_{b, a}\right) \geq T_{a} \\
0, \text { если }\left|W_{\Psi} f_{b, a}\right|<T_{a} \\
-W_{\Psi} f_{b, a}, \text { если }\left(W_{\Psi} f_{b, a}\right)<-T_{a}
\end{array},\right.
$$

where $T_{a}=U * S t^{l}{ }_{a}$ is the threshold function where $S t^{l}{ }_{a}=\sqrt{\frac{1}{l-1} \sum_{k=1}^{l}\left(W_{\Psi} f_{b, a}-\overline{W_{\Psi} f_{b, a}}\right)^{2}}$, is the standard deviation, $l$ is the time window length, $\overline{W_{\Psi} f_{b, a}}$ is the average value, $U$ is the threshold coefficient.

It is obvious that the parameters of function (5), the window length $l$ and the threshold coefficient $U$, are adjustable and determine the size of a time window within which geomagnetic disturbances are estimated and the level of determined geomagnetic disturbances (we applied the window length of $l=1440$ that corresponds to 24 hours and the threshold coefficient $U=7$ ).

To estimate the intensity of the detected disturbances at an instant time $t=b$ according to the paper [30], we apply the value of

$$
Y_{b}=\sum_{a} P_{T_{a}}\left(W_{\Psi} f_{b, a}\right)
$$

We make wavelet transform of value $Y_{b}$ (see (4))

$$
\left(W_{\Psi} Y_{c, d}\right):=|d|^{-1 / 2} \int_{-\infty}^{\infty} f(t) \Psi\left(\frac{t-c}{d}\right) d t, d, c \in R, d \neq 0,
$$

and taking into account that a wavelet is a window function [31], we obtain a dynamic spectrum of geomagnetic disturbance intensity.

\section{Processing results of geomagnetic data during the magnetic storms on January 7, 2015 and March 17, 2015}

Based on the suggested method, we processed and analyzed the data from the sites in the north-eastern segment of Russia (Table 1). To analyze the processes in the magnetosphere at the near equatorial latitudes, the data of the Indian HYB "Hydarabad" and CPL "Choutuppal" sites were used. The considered events and the results of application of the developed method for detection of anomalous increases of geomagnetic disturbance intensity before magnetic storms is shown in Table 2 . Analysis of the results of Table 2 indicates the possibility of occurrence of weak geomagnetic disturbances before magnetic storms, which was first mentioned in the papers [33,34]. It shows high sensitivity and the efficiency of the method suggested in the paper. In what follows are the detailed results of geomagnetic data processing during geomagnetic storms which occurred on 07.01.2015, 17.03.2015, 21.06.2015, 15.08.2015, 19.12.2015.

\begin{tabular}{lllllll}
\multicolumn{2}{l}{ Table 1. Sites of the north-eastern segment of Russia. } \\
\hline Observatory & $\begin{array}{l}\text { Code } \\
\text { IAGA }\end{array}$ & $\begin{array}{l}\text { Geographical } \\
\text { latitude }\end{array}$ & $\begin{array}{l}\text { Geographical } \\
\text { longitude }\end{array}$ & $\begin{array}{l}\text { Geomagnetic } \\
\text { latitude }\end{array}$ & $\begin{array}{l}\text { Geomagnetic } \\
\text { longitude }\end{array}$ & $\begin{array}{l}\text { Local time } \\
\text { (LT) }\end{array}$ \\
\hline Magadan (1) & MGD & $59^{\circ} 33.1^{\prime}$ & $150^{\circ} 48.3^{\prime}$ & $51^{\circ} 32.4^{\prime}$ & $146^{\circ} 2.4^{\prime}$ & UTC+11 \\
Paratunka (1) & PET & $52^{\circ} 58.3^{\prime}$ & $158^{\circ} 15.0^{\prime}$ & $45^{\circ} 51.6^{\prime}$ & $137^{\circ} 57.6^{\prime}$ & UTC+12 \\
Khabarovsk (1) & KHB & $48^{\circ} 29.0^{\prime}$ & $135^{\circ} 04.0^{\prime}$ & $39^{\circ} 15^{\prime}$ & $156^{\circ} 48.6^{\prime}$ & UTC+10 \\
Choutuppal (2) & CPL & $17^{\circ} 17.33^{\prime}$ & $78^{\circ} 55^{\prime}$ & $8^{\circ} 37.2^{\prime}$ & $152^{\circ} 34.8^{\prime}$ & UTC+5:30 \\
\hline
\end{tabular}

Note: site affiliation is indicated in brackets (1) - IKIR FEB RAS, (2) - CSIR-National Geophysical Research Institute. 
Image Processing, Geoinformation Technology and Information Security / O. Mandrikova et al.

Table 2. Results of detection of anomalous increases of geomagnetic activity before storms.

\begin{tabular}{|c|c|c|c|c|c|c|c|c|}
\hline \multirow[t]{2}{*}{$\begin{array}{l}\text { Date of a } \\
\text { storm }\end{array}$} & \multirow[t]{2}{*}{ Storm source } & \multirow[t]{2}{*}{$\begin{array}{l}\text { Time of storm } \\
\text { beginning (UT) }\end{array}$} & \multirow[t]{2}{*}{$\begin{array}{l}\max \\
\mathrm{Kp}\end{array}$} & \multirow[t]{2}{*}{$\begin{array}{l}\max \\
\text { Dst }\end{array}$} & \multicolumn{2}{|c|}{$\begin{array}{l}\text { Anomalies detected before a magnetic } \\
\text { storm }\end{array}$} & \multicolumn{2}{|c|}{$\begin{array}{l}\text { Maximum value of the } \\
\text { detected disturbance } \\
\text { intensities }\end{array}$} \\
\hline & & & & & $\begin{array}{l}\text { Time interval before a } \\
\text { magnetic storm } \\
\text { beginning }\end{array}$ & Scales & KHB site & CPL site \\
\hline \multirow[t]{3}{*}{07.01 .2015} & $\mathrm{CME}$ & $6: 15$ & 6 & -103 & 4 hours 55 minutes & $4-10$ & 20 & 0 \\
\hline & & & & & 12 hours 10 minutes & $2-8$ & 12 & 27 \\
\hline & & & & & 21 hours & $14-20$ & 1085 & 328 \\
\hline \multirow[t]{3}{*}{ 17.03.2015 } & CME & $4: 45$ & 8 & -233 & 11 hours 15 minutes & $5-40$ & 523 & 225 \\
\hline & & & & & 12 hours 10 minutes & $20-34$ & 232 & 0 \\
\hline & & & & & 16 hours 30 minutes & $6-10$ & 3 & 2 \\
\hline \multirow[t]{2}{*}{21.06 .2015} & CIR/CME & $16: 55$ & 8 & -111 & 7 hours & $10-16$ & 2 & 53 \\
\hline & & & & & 10 hours & $0-8$ & 2 & 0 \\
\hline \multirow[t]{7}{*}{15.08 .2015} & CME/CIR & $8: 30$ & 6 & -64 & 5 hours & $8-12$ & 0 & 209 \\
\hline & & & & & 5 hours 20 minutes & $40-60$ & 2 & 415 \\
\hline & & & & & 5 hours 30 minutes & $8-20$ & 65 & 35 \\
\hline & & & & & 7 hours & $0-8$ & 3 & 0 \\
\hline & & & & & 10 hours & $16-32$ & 1 & 0 \\
\hline & & & & & 13 hours 30 minutes & $8-22$ & 18 & 19 \\
\hline & & & & & 18 hours 40 minutes & $0-4$ & 1 & 0 \\
\hline \multirow[t]{4}{*}{ 19.12.2015 } & CME & $16: 18$ & 7 & -170 & 1 hour & $8-32$ & 1 & 0 \\
\hline & & & & & 7 hours 20 minutes & $6-12$ & 4 & 0 \\
\hline & & & & & 15 hours 30 minutes & $2-8$ & 5 & 0 \\
\hline & & & & & 25 hours 30 minutes & $0-4$ & 1 & 1 \\
\hline
\end{tabular}

a)

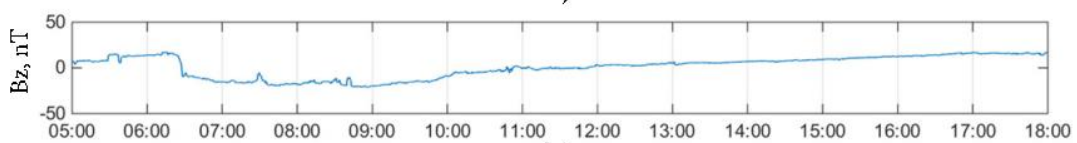

b)

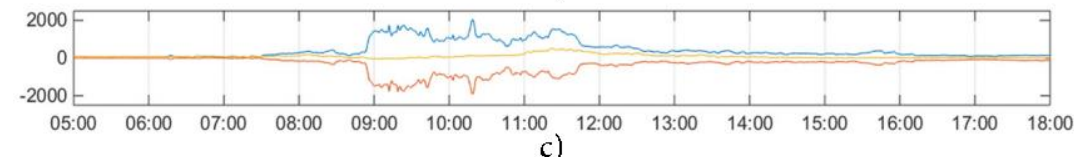

c)

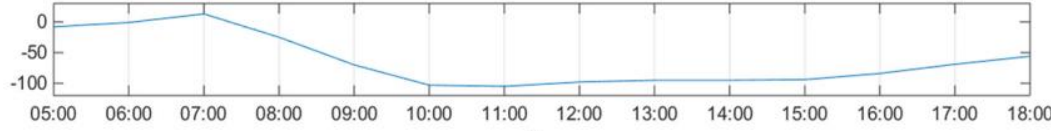

d)
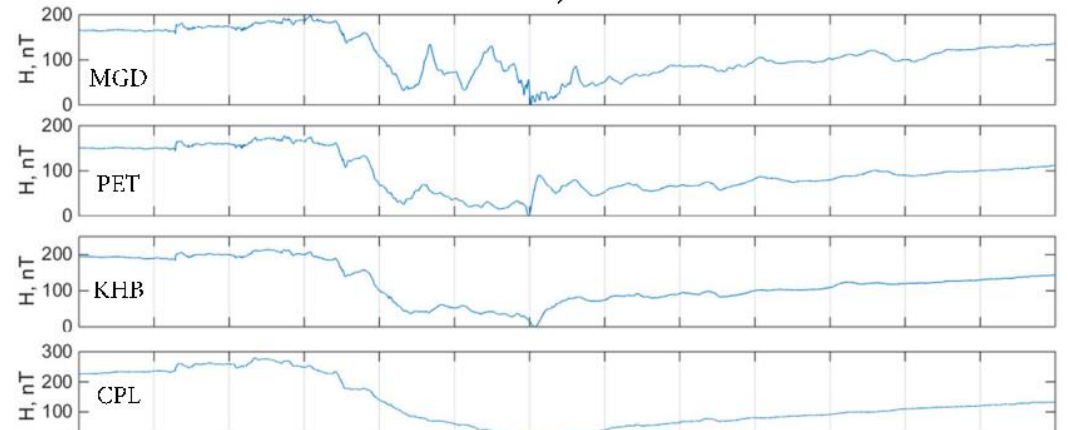

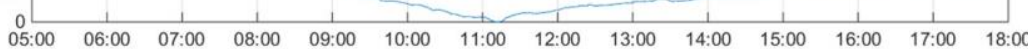

e)

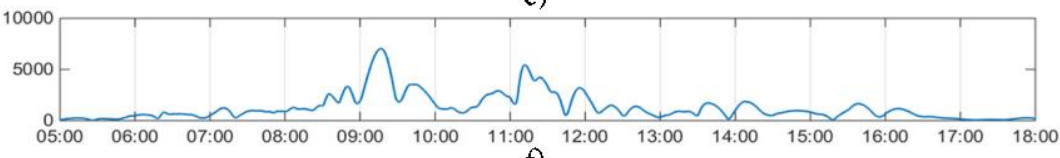

f)

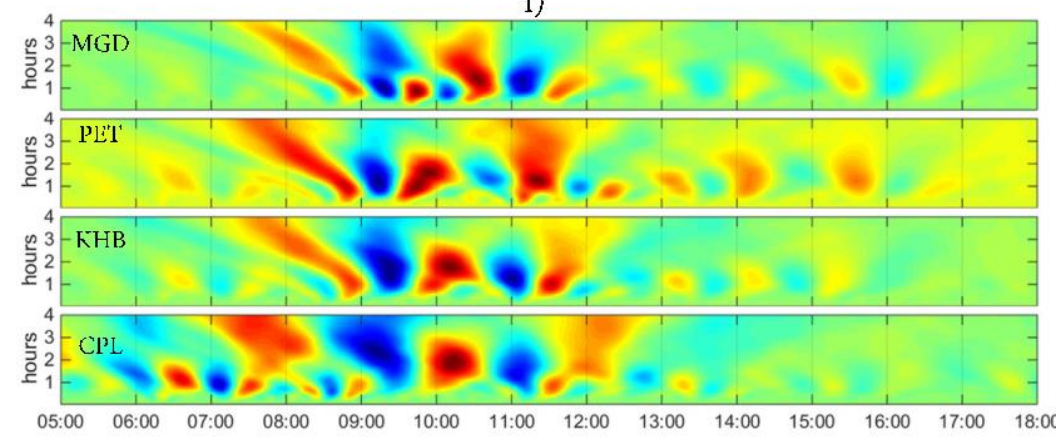

Fig 1. Processing results of the data for January 7, 2015; a) Bz component of the Interplanetary Magnetic Field; b) AE-index (yellow line), AU-index (blue line) and AL-index (red line); c) Dst-index; d) H-component of the magnetic field; e) geomagnetic disturbance intensity (relation (5)); f) dynamic spectrum of geomagnetic disturbance intensity. 
a)

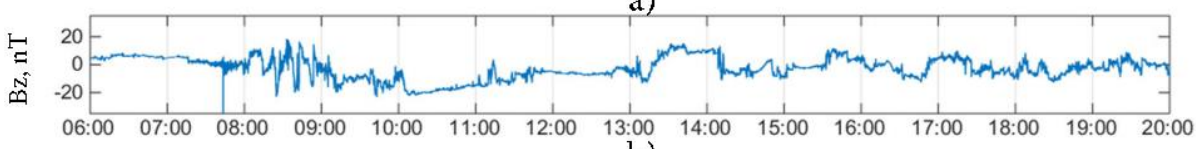

b)
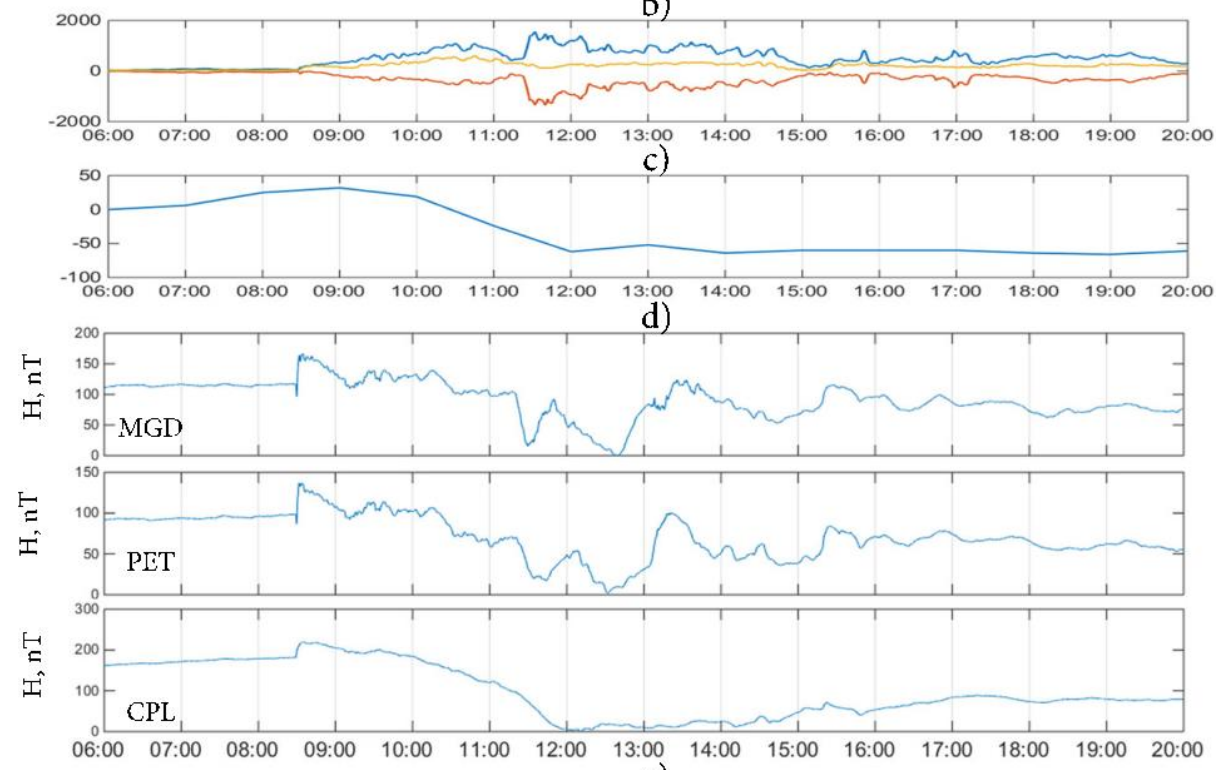

e)

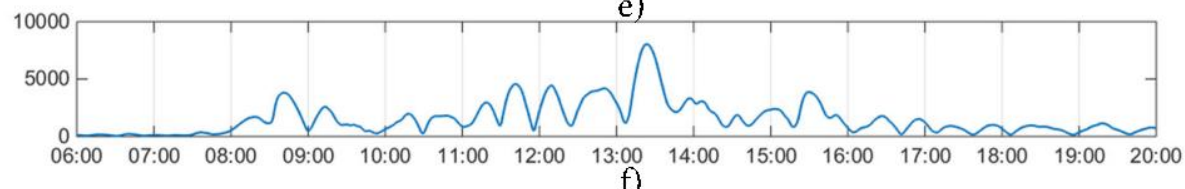

f)
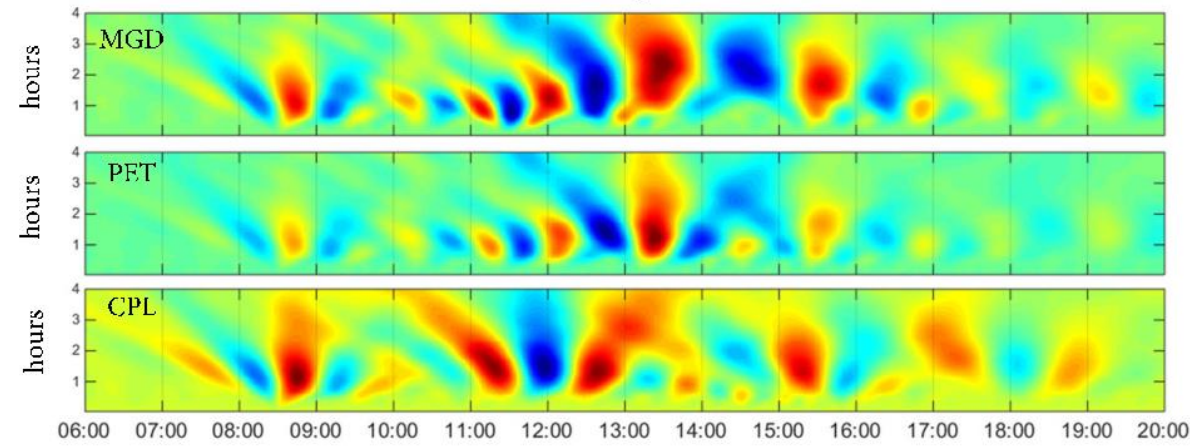

Fig. 2. Processing results of the data for August 15, 2015; a) Bz component of the Interplanetary Magnetic Field; b) AE-index (yellow line), AU-index (blue line) and AL-index (red line); c) Dst-index; d) H-component of the magnetic field; e) geomagnetic disturbance intensity (relation (5)); f) dynamic spectrum of geomagnetic disturbance intensity.

Fig. 1 shows the results of processing of geomagnetic data during the magnetic storm on January 7, 2015. This event was caused by coronal ejection of solar material (CME on January 4, http://ipg.geospace.ru/space-weather-review-07-01-2015.html). Its dynamics was of classical character with clearly defined major phases of a storm in Dst-variation (Fig. 1c). The results of estimation the geomagnetic disturbance intensity shows that during the initial stage of the storm, from about 07:00 UT, geomagnetic activity gradually increased and the Dst-index had positive values. Maxima of disturbance intensity (Fig. 1e) are observed during Dst-index decrease and AE-index increase characterizing the occurrence of an intensive substorm in the auroral zone. The dynamic spectrum of geomagnetic disturbance intensity (relation (5)) illustrated in Fig. 1f shows the regions of disturbance concentration and propagation in the areas under analysis. During the event, a general picture of the dynamics of magnetospheric current systems is observed. The beginning of the storm from 6:00 to 08:00 UT was the most clearly defined at the near equatorial site (India). During the main phase of the storm, activation areas are observed in the dynamic spectra of all the sites (Fig. 1f; red color is the intensity increase; blue color is the intensity decrease). They are likely to characterize largescale processes in the magnetosphere probably associated with energy accumulation and release during the event. At the most northern site Magadan, local regions (from 10:00 to 11:00 UT) are distinguished. They are likely to be associated with auroral processes.

Fig. 2 shows the data processing for the magnetic storm on August 15, 2015 which was caused by a solar medium coronal mass ejection (CME on August 12) and high-velocity flows from a coronal hole (CIR). The magnetic storm began at 08:30 UT during a sharp increase of solar wind velocity and increase of the magnetic field horizontal component at all the sites under analysis. Short-period anomalous increases of geomagnetic activity began about 12 hours before the magnetic storm (Fig. 2e). The highest values of geomagnetic disturbance intensity are observed at the sites during the main phase of the storm (the period of significant decrease of Dst-indes). The wavelet spectrum of geomagnetic disturbance intensity shows that geomagnetic field 
disturbances at all the sites increased in the vicinity of special points (points of local extreme periods, function inflection) of Dst-variation (08:00-10:00; 11:00-13:00 UT). It indicates active processes in the magnetosphere at these time periods. The disturbances at the near equatorial site CPL had the most clearly defined character.

The results of application of the developed method for detection of pre-storm anomalies of geomagnetic disturbance intensity increases are illustrated on the example of the event on August 15, 2015 in Fig. 3. Analysis of Fig. 3e shows synchronous anomalous increases of geomagnetic activity 18 hours before the magnetic storm. Several minutes before registration of the event at the sites, short-time geomagnetic disturbance intensity significantly increased and reached the maximum values during the initial phase.

a)

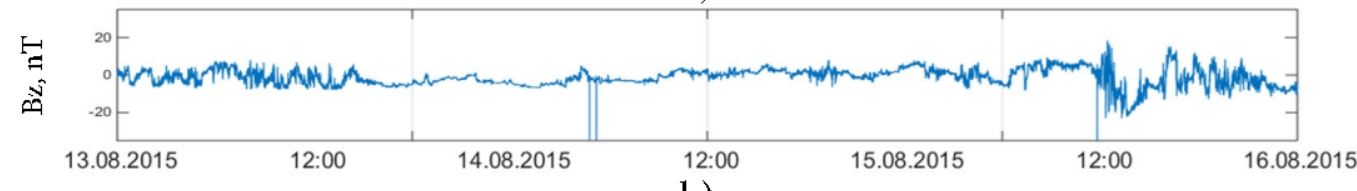

b)

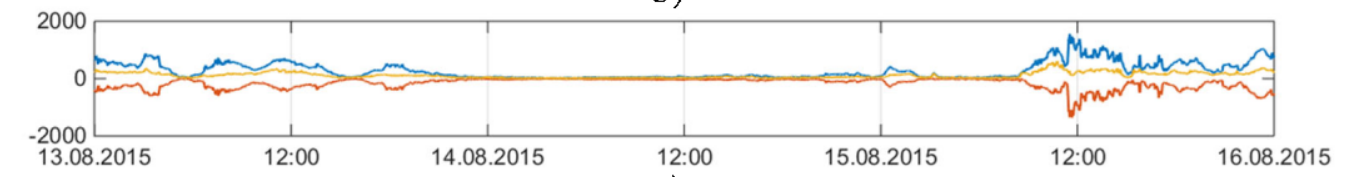

c)

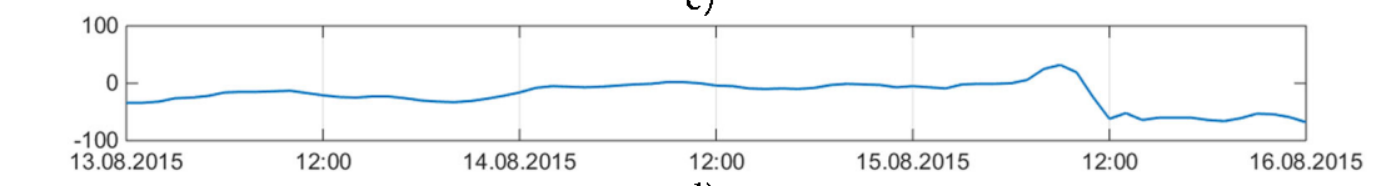

d)

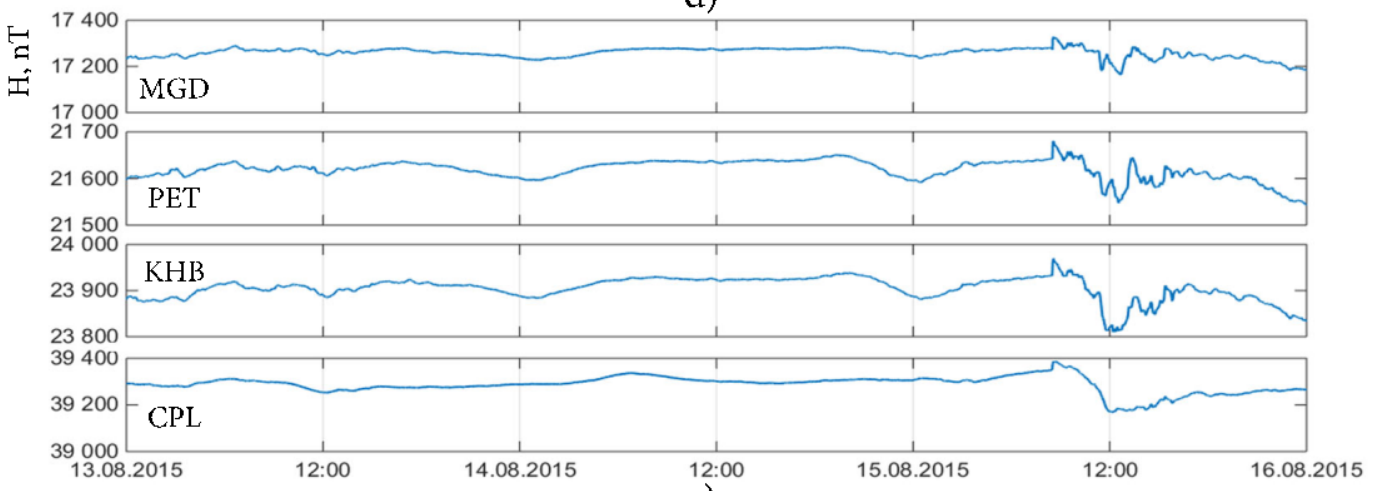

e)

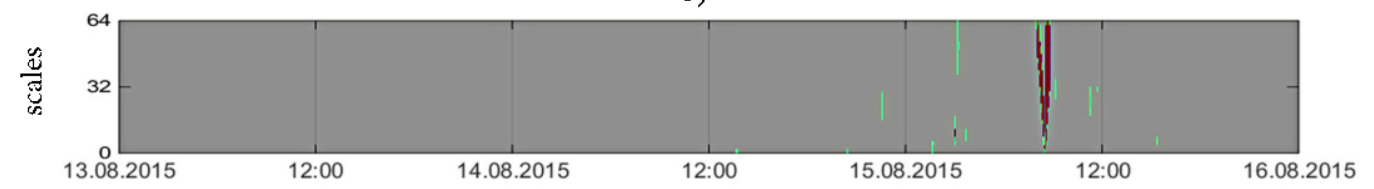

Fig. 3. Processing results of the data for August 13-15, 2015; a) Bz component of the Interplanetary Magnetic Field; b) AE-index (yellow line), AU-index (blue line) and AL-index (red line); c) Dst-index; d) H-component of the magnetic field; e) detection of the periods of increased geomagnetic activity (relation

\section{Conclusions}

(6)).

A detailed analysis of geomagnetic data during strong magnetic storms in 2015 was carried out by the suggested method. The dynamic spectrum of geomagnetic disturbance intensity showed spatial pattern of the events and allowed us to analyze geomagnetic disturbance propagation along the observation meridian and at the near equatorial sites. During the main phases of the storms, activation areas were detected. They have large spatial scales and are likely to be associated with the processes of energy accumulation and release in the magnetosphere. Before the events, synchronous local increases of geomagnetic activity were observed at the sites under analysis. They are likely to be associated with nonstationary effect of solar wind plasma on the Earth magnetosphere in the course of an interplanetary disturbance approaching. Such anomalous pre-storm effects are mentioned in the papers [33,34]. According to the processing results of large experimental material and joint analysis of geomagnetic field H-component oscillations with the oscillating processes on the Sun, the authors [13, 33] showed that the success rate of the suggested forecast method for the geoeffective flare events is $90 \%$. This result indicates high probability of possible occurrence of pre-storm anomalous features in geomagnetic data. The possibility of automatic registration of anomalous feature data is an important aspect of the suggested method for space weather forecast.

\section{Acknowledgments}

The development of the method for geomagnetic data analysis was supported by RSF Grant № 14-11-00194. The data primary analysis was supported by RFBR Grant № 16-55-45007. The authors are grateful to the Institutes supporting the magnetic observatories which data were used in the investigation. 


\section{References}

[1] Yermolaev YuI, Yermolaev MYu. Solar and Interplanetary Sources of Geomagnetic Storms: Space Weather Aspects. Izvestiya, Atmospheric and Oceanic Physics 2010; 46(7): 799-819.

[2] Nayar SRP, Radhika VN, Seena PT. Investigation of substorms during geomagnetic storms using wavelet techniques. Proceedings of the ILWS Workshop Goa, India, 2006: 328-331.

[3] Hafez AG, Ghamry E, Yayama H, Yumoto K. Systematic examination of the geomagnetic storm sudden commencement using multi resolution analysis. Advances in Space Research 2013; 51: 39-49.

[4] Xu Z, Zhu L, Sojka J, Kokoszka P, Jach A. An assessment study of the wavelet-based index of magnetic storm activity (WISA) and its comparison to the Dst index. J. Atmos. Solar-Terr. Phys. 2008; 70: 1579-1588.

[5] Jach A, Kokoszka P, Sojka J, Zhu L. Wavelet-based index of magnetic storm activity. J. Geophys. Res. 2006; 111(A9). DOI:10.1029/2006JA011635.

[6] Paschalis P, Sarlanis C, Mavromichalaki H. Artificial neural network approach of cosmic ray primary data processing. Solar Physics 2013; 182(1): 303318.

[7] Macpherson KP, Conway AJ, Brown JC. Prediction of solar and geomagnetic activity data using neural networks. J. Geophys. Res. 2001 ; 100 : 735-744.

[8] Woolley JW, Agarwarl PK, Baker J. Modeling and prediction of chaotic systems with artificial neural networks. International Journal for Numerical Methods in Fluids 2010; 63. DOI:10.1002/fld.2117.

[9] Soloviev A, Chulliat A, Bogoutdinov S, Gvishiani A, Agayan S, Peltier A, Heumez B. Automated recognition of spikes in 1 Hz data recorded at the Easter Island magnetic observatory. Earth Planets Space 2012; 64(9): 743-752.

[10] Rotanova N, Bondar T, Ivanov V. Wavelet Analysis of Secular Geomagnetic Variations. Geomagnetism and Aeronomy 2004; $44: 252-258$.

[11]Rybàk J, Antalovà A, Storini M. The wavelet analysis of the solar and cosmic-ray data. Space Science Reviews 2001; 97: 359-362.

[12]Zaourar N, Hamoudi M, Mandea M, Balasis G, Holschneider M. Wavelet-based multiscale analysis of geomagnetic disturbance. Earth Planets Space 2013; 65(12): 1525-1540.

[13] Smirnova AS, Snegirev SD, Sheyner OA. Sun ultraviolet radiation as a possible cause of preflare long-period oscillations of geomagnetic field horizontal component. Vestnik of Lobachevsky University of Nizhni Novgorod 2013; 6(1): 88-93.

[14] Mandrikova OV, Solovev IS, Zalyaev TL. Methods of analysis of geomagnetic field variations and cosmic ray data. Earth Planet Space 2014 ; 66. DOI:10.1186/s40623-014-0148-0.

[15] Golovkov VP, Papitashvili VO, Papitashvili NE. Automated calculation of the K indices using the method of natural orthogonal components, Geomagn. Aeron 1989; 29: 667-670.

[16] Nowożyński K, Ernst T, Jankowski J. Adaptive smoothing method for computer derivation of K-indices. Geophys. J. Int. 1991 ; 104 : 85-93.

[17] Menvielle M, Papitashvili N, Hakkinen L, Sucksdorff C. Computer production of K indices: review and comparison of methods. Geophys. J. Int. 1995; 123: 866-886.

[18] Mandrikova OV, Smirnov SE, Solov'ev IS. Method for Determining the Geomagnetic Activity Index Based on Wavelet Packets. Geomagnetism and Aeronomy 2012; 52(1): 111-120.

[19] Bogoutdinov SR, Gvishiani AD, Agayan SM, Solovyev AA, Kihn E. Recognition of Disturbances with Specified Morphology in Time Series. Part 1: Spikes on Magnetograms of the Worldwide INTERMAGNET Network. Izvestiya, Physics of the Solid Earth 2010; 46(11): 1004-1016.

[20] Sidorov RV, Soloviev AA, Bogoutdinov ShR. Application of the SP algorithm to the INTERMAGNET magnetograms of the disturbed geomagnetic field. Izvestiya, Physics of the Solid Earth 2012; 48(5): 410-414.

[21] Krasnoperov RI, Soloviev AA. Analytical geoinformation system for integrated geological-geophysical research in the territory of Russia. GornyiZhurnal 2015; 10: 89-93. DOI: 10.17580/gzh.2015.10.16.

[22] Soloviev A. et al. Data Science Journal 2013; 12. DOI:10.2481/dsj.WDS-019.

[23] Uwamahoro J, McKinnell LA, Habarulema JB. Estimating the geoeffectiveness of halo CMEs fro mass ociated solar and IP parameters using neural networks. Annales Geophysicae 2012; 30: 963-972.

[24] Mahrous A, Radi A, Youssef M, Faheem A, Ahmed S, Gopalswamy N. Prediction of the interplanetary Coronal Mass Ejection and it's associated shock by using neural network. 38th COSPAR Scientific Assembly in Bremen, Germany 2010; D23-0052-10.

[25] Pallocchia G, Amata E, Consolini G, Marcucci MF, Bertello I. Geomagnetic Dst index forecast based on IMF data only. Annales Geophysicae 2006; 24: 989-999. URL: www.ann-geophys.net/24/989/2006/.

[26] Mandrikova OV. Multicomponent model of a signal with a complicated structure. Problems of the evolution of open systems 2008; 2(10): 161-172. (in Russian)

[27] Mandrikova OV, Polozov YuA. Approximation and analysis of ionospheric parameter based on the combination of wavelet transform and neural network collectives. Information technologies 2014; 7: 61-65. (in Russian)

[28] Geppener VV, Mandrikova OV, Zhizhikina EA. Automatic method for estimation of the earth's magnetic field state. Proceedings of international conference on soft computing and measurements, SCM 2015; 18: 251-254. DOI: 10.1109/scm.2015.7190473.

[29] Mandrikova OV, Zhizhikina EA. An automatic method for estimating the geomagnetic field. Computer Optics 2015; 39(3): 420-428. DOI: $10.18287 / 0134-2452-2015-39-3-420-428$.

[30] Mandrikova OV, Solovjev I, Geppenerc V, Taha A-KR, Klionskiy D. Analysis of the Earth's magnetic field variations on the basis of a wavelet-based approach. Digit Signal Process 2013; 23: 329-339.

[31] Chui CK. An introduction in wavelets. Academic Press, New York, 1992; 264 p.

[32] Daubechies I. Ten Lectures on Wavelets. CBMS-NSF Lecture Notes 1992; 61: 377 p.

[33] Sheiner OA, Fridman VM. The features of microwave solar radiation observed in the stage of formation and initial propagation of geoeffective coronal mass ejections. Radiophysics and Quantum Electronics 2012; 54(10): 655-666.

[34] Mandrikova OV, Bogdanov VV, Solov'ev IS. Wavelet analysis of geomagnetic field data. Geomagnetism and Aeronomy 2013 ; 53(2): $268-273$. 